\title{
Serum uric acid is associated with increased risk of idiopathic venous thromboembolism in high HDL-C population: A case-control study
}

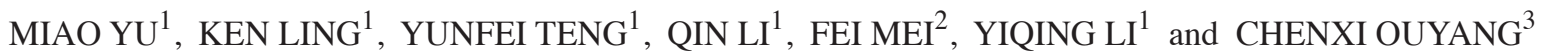 \\ ${ }^{1}$ Department of Vascular Surgery, Wuhan Union Hospital, Tongji Medical College, Huazhong \\ University of Science and Technology, Wuhan, Hubei 430022; ${ }^{2}$ Department of Vascular Surgery, Yichang \\ Central People's Hospital, The First College of Clinical Medical Sciences, Three Gorges University, Yichang, \\ Hubei 443003; ${ }^{3}$ Department of Vascular Surgery, Fuwai Hospital, National Center for Cardiovascular Disease, \\ Chinese Academy of Medical Sciences and Peking Union Medical College, Beijing 100037, P.R. China
}

Received February 25, 2016; Accepted April 4, 2016

DOI: $10.3892 /$ etm.2016.3228

\begin{abstract}
Many studies have indicated that metabolic disorders are positively correlated with idiopathic venous thromboembolism (VTE), whereas the risk factor serum uric acid (SUA) for idiopathic VTE has yet to be investigated. In this retrospective case-control study, 276 idiopathic VTE patients and 536 gender- and age-matched control subjects were included. The subjects in the case and control groups exhibiting common known VTE risk factors and the patients with a first VTE onset in one month were excluded. For the control group, primary and secondary VTE patients were excluded. High-density lipoprotein cholesterol (HDL-C), low-density lipoprotein cholesterol, total cholesterol, fasting blood glucose, and current smoking were significantly associated with idiopathic VTE in the univariate analysis. Hyperuricemia was detected in 56/276 (20.29\%) idiopathic patients compared with $71 / 536(13.25 \%)$ in the control group. HDL-C was considered the most prominent interactive factor for SUA in idiopathic VTE by the interaction analysis. After testing for the interaction terms, SUA was closely associated with idiopathic VTE in the high HDL-C population ( $\mathrm{P}=0.0026$ for interaction), while there was no such correlation in the low HDL-C group. The results indicated no
\end{abstract}

Correspondence to: Dr Chenxi Ouyang, Department of Vascular Surgery, Fuwai Hospital, National Center for Cardiovascular Disease, Chinese Academy of Medical Sciences and Peking Union Medical College, 167 Beilishi Road, Beijing 100037, P.R. China E-mail: chenxi_ouyang1@163.com

Dr Yiqing Li, Department of Vascular Surgery, Wuhan Union Hospital, Tongji Medical College, Huazhong University of Science and Technology, 1277 Jiefang Street, Wuhan, Hubei 430022, P.R. China E-mail: yiqing_li1@163.com

Key words: serum uric acid, high-density lipoprotein cholesterol, idiopathic venous thromboembolism, interaction analysis obvious correlation between triglyceride and hypertension to idiopathic VTE. In conclusion, SUA is closely associated with an increased risk of idiopathic VTE in the high HDL-C population. The abnormality of SUA may act as an important linkage between atherosclerosis and idiopathic VTE through HDL-C.

\section{Introduction}

Venous thromboembolism (VTE) is a common disease with high prevalence and various etiological factors. In developed countries, the annual incidence of VTE is $\sim 1-2$ events/1,000 individuals (1). Surgery, fracture, malignancy, immobilization, elevated homocystein, protein $\mathrm{C}$-, protein $\mathrm{S}$ - or antithrombin-deficiency, and prothrombin G20210A variation are considered established risk factors for VTE (1). However, a large number of patients develop VTE without specific reasons and are regarded as idiopathic VTE patients who comprise $>30 \%$ of the total VTE events (2).

Arterial thromboemboli are white thrombi that exist in the high shear and platelet-rich area, while venous thrombosis are red thrombi that are generally characterized by red cells under hypercoagulation and venous stasis (3). The thrombi are considered independent entities with different pathological mechanisms and therapeutic consequences. However, since idiopathic VTE and atherosclerotic vascular disorders may share some common risk factors, obesity, dyslipidemia, metabolic syndrome, smoking, hypertension, estrogen and other risk factors have been investigated to determine the association. Findings of previous studies $(4,5)$ on obesity, metabolic syndrome and high-density lipoprotein cholesterol (HDL-C) are inconsistent, with metabolic syndrome and HDL-C being the most controversial. Ageno et al (6) conducted a meta-analysis indicating an obvious correlation between low HDL-C levels, high triglyceride levels and VTE. A randomized trial (JUPITER trial) showed that rosuvastatin significantly reduced the occurrence of symptomatic VTE in a healthy population (7). However, two large population-based prospective studies demonstrated 
that metabolic syndrome, diabetes, hypertension and other traditional atherosclerotic risk factors did not contribute to VTE but that only abdominal obesity was relevant $(4,8)$.

Besides dyslipidemia, hyperglycemia and hypertension, hyperuricemia has been considered an independent risk factor for cardiovascular disease (CVD) (9), although it is rarely associated with venous thrombosis. A retrospective case-control study was therefore conducted to investigate the correlation of serum uric acid (SUA) and idiopathic VTE in a Chinese population. In the hospital-based case-control study, we focused on the risk of idiopathic VTE correlated with SUA, as well as blood lipids, fasting blood glucose (FBG), current smoking status and hypertension.

\section{Patients and methods}

Patients and diagnostic criteria. The study was conducted between January 2012 and June 2015, and 1,147 VTE patients treated in the Department of Vascular Surgery (Union Hospital, Tongji Medical College, Huazhong University of Science and Technology, Wuhan, China) were enrolled. Six patients were excluded due to age restriction ( $<20$ years). In addition, 845 patients were excluded based on the exclusion criteria mentioned below. After checking for the integrity of the data, there were 12 patients without SUA, 7 patients without blood lipids and 1 patient without ultrasonography information; these patients were also excluded. A total of 276 patients diagnosed as idiopathic VTE with objective diagnosis of deep vein thrombosis (DVT) or pulmonary embolism (PE) were included in the study. Intervals carried out from clinical onset to diagnosis occurred within 1 month. DVT was diagnosed by color Doppler ultrasonography and PE was diagnosed by computed tomography angiography (CTA). Idiopathic VTE was defined as the absence of risk factors including surgery, trauma, pregnancy, delivery, cachexia, immobilization, malignancy, secondary VTE, use of oral contraceptives, transsexualism, mental disorders and known hypercoagulable disorders. As many as 4,632 control subjects with the same ethnic background from the Physical Examination Center of Union Hospital, Tongji Medical College, Huazhong University of Science and Technology were included in the study. However, 974 subjects, aged $<20$ years were excluded, as well as 894 men and 963 women. After randomly matching for gender and age at a ratio of 1:2,305 males and 231 females served as the control group. The exclusion criteria for the control group included surgery, trauma, pregnancy, delivery, cachexia, immobilization, malignancy, use of oral contraceptives, transsexualism, mental disorders, arterial or VTE and known hypercoagulable disorders (Fig. 1).

Data collection. The following clinical data were collected from the patients and controls: Age, gender, site of thrombosis, smoking status, blood pressure, serum levels of SUA, HDL-C, low-density lipoprotein cholesterol (LDL-C), triglyceride, total cholesterol, and FBG. Hyperuricemia was defined as SUA $>420 \mu \mathrm{mol} / 1(7.0 \mathrm{mg} / \mathrm{dl})$ for male or $>357 \mu \mathrm{mol} / 1(6.0 \mathrm{mg} / \mathrm{dl})$ for female patients. Dyslipidemia was defined as HDL-C $<1.036 \mathrm{mmol} / 1$ (40 mg/dl) for male or $<1.295 \mathrm{mmol} / 1$ (50 mg/dl) for female patients, and/or LDL-C >3.1 mmol/1 (120 mg/dl), and/or total cholesterol
Table I. Characteristics of participants with or without an idiopathic VTE.

\begin{tabular}{lccr}
\hline Characteristics & $\begin{array}{c}\text { Idiopathic } \\
\text { VTE }(\mathrm{n}=276)\end{array}$ & $\begin{array}{c}\text { Control } \\
(\mathrm{n}=536)\end{array}$ & P-value \\
\hline Age (years) & $52.9 \pm 14.4$ & $52.7 \pm 14.5$ & 0.901 \\
Man & $158(57.2 \%)$ & $309(57.6 \%)$ & 0.912 \\
HDL-C (mmol/l) & $1.30 \pm 0.38$ & $1.36 \pm 0.32$ & 0.028 \\
LDL-C (mmol/l) & $2.60 \pm 0.79$ & $2.40 \pm 0.74$ & $<0.001$ \\
Triglyceride & $1.21(0.88-1.66)$ & $1.14(0.83-1.59)$ & 0.066 \\
(mmol/l) & & & \\
Total cholesterol & $4.58 \pm 0.98$ & $4.39 \pm 0.94$ & 0.006 \\
(mmol/l) & & & \\
FBG (mmol/l) & $5.76 \pm 1.84$ & $5.21 \pm 1.44$ & $<0.001$ \\
SUA ( $\mu$ mol/l) & $320.5 \pm 101.3$ & $309.8 \pm 87.9$ & 0.121 \\
Current smoker & $83(30.1 \%)$ & $128(23.6 \%)$ & 0.057 \\
Hypertension & $44(15.9 \%)$ & $82(15.3 \%)$ & 0.810 \\
\hline
\end{tabular}

The descriptive measures were expressed as frequency and proportion for categorical variables, and mean \pm standard deviations (SD) or median and interquartile for continuous variables. The differences between groups were analyzed using the Chi-square test for categorical variables and the t-test (normal distribution) or Kruskal-Wallis rank sum test (non-normal distribution) for continuous variables. HDL-C, high-density lipoprotein cholesterol; LDL-C, low-density lipoprotein cholesterol; VTE, venous thromboembolism; SUA, serum uric acid; FBG, fasting blood glucose.

$>5.18 \mathrm{mmol} / \mathrm{l}(200 \mathrm{mg} / \mathrm{dl})$, and/or triglyceride $>1.7 \mathrm{mmol} / \mathrm{l}$ (150 mg/dl). Hyperglycemia was defined as FBG >6.1 mmol/1 (110 mg/dl). Smoking status was divided into current smoker or non-smoker, and hypertension was defined as systolic blood pressure $>140 \mathrm{mmHg}$ and/or diastolic blood pressure $>90 \mathrm{mmHg}$. HDL-C, total cholesterol, triglyceride and FBG were determined in fresh plasma. The LDL-C was measured by the Friedewald equation except for patients with plasma triglycerides $>400 \mathrm{mg} / \mathrm{dl}$. FBG was tested after $6 \mathrm{~h}$ of fasting. After $10 \mathrm{~min}$ rest, blood pressure was measured in the patient's right radial artery in the sitting position using an electronic sphygmomanometer. Measurements were repeated after 5 and $10 \mathrm{~min}$ from the initial measurement. The mean value of the three measurements was recorded. Age for control subjects was matched \pm 2 years for idiopathic VTE patients.

Statistical analysis. Group differences were examined with regard to gender, age (years), HDL-C (mmol/l), LDL-C $(\mathrm{mmol} / \mathrm{l})$, triglyceride $(\mathrm{mmol} / \mathrm{l})$, total cholesterol $(\mathrm{mmol} / \mathrm{l})$, FBG (mmol/l), SUA ( $\mu \mathrm{mol} / \mathrm{l})$, current smoker (yes or no), and hypertension (yes or no) in idiopathic VTE patients. The descriptive measures were expressed as frequency and proportion for categorical variables, and mean \pm standard deviations (SDs) or median and interquartile for continuous variables. The differences between groups were analyzed using the Chi-square test for categorical variables and the t-test (normal distribution) or Kruskal-Wallis rank sum test (non-normal distribution) for continuous variables (Table I). 
Idiopathic VTE group

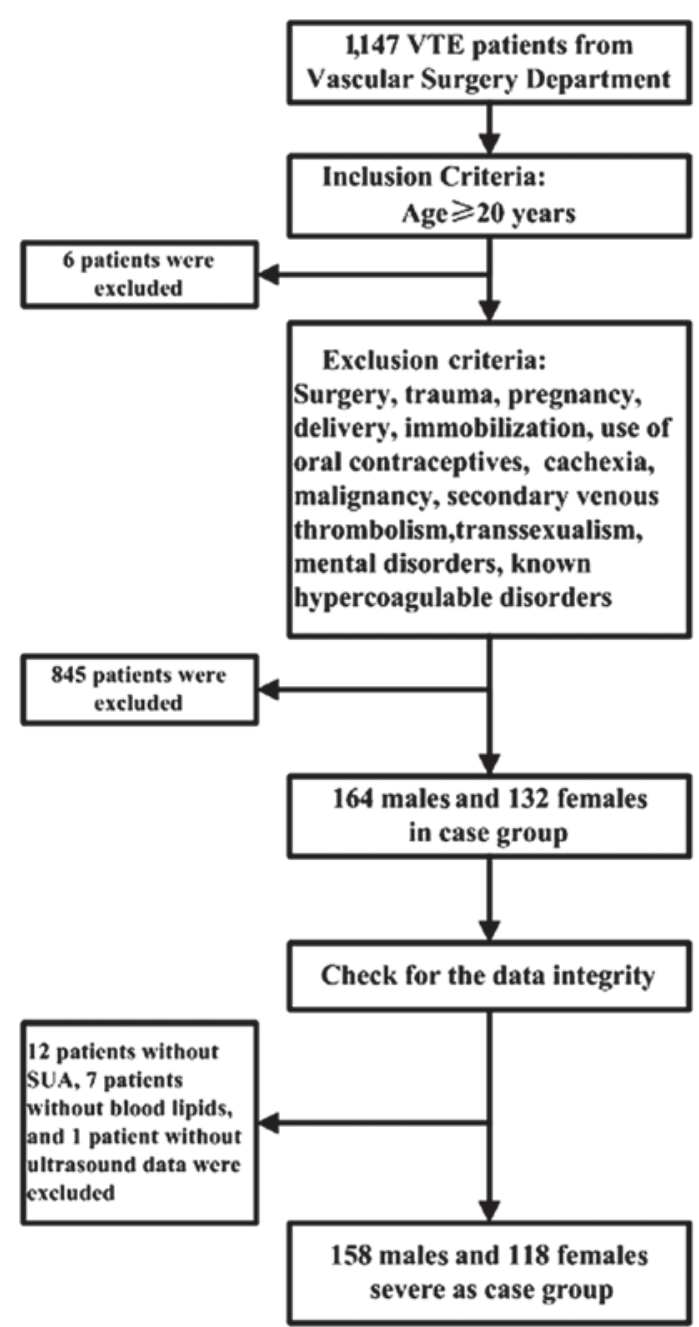

Control group

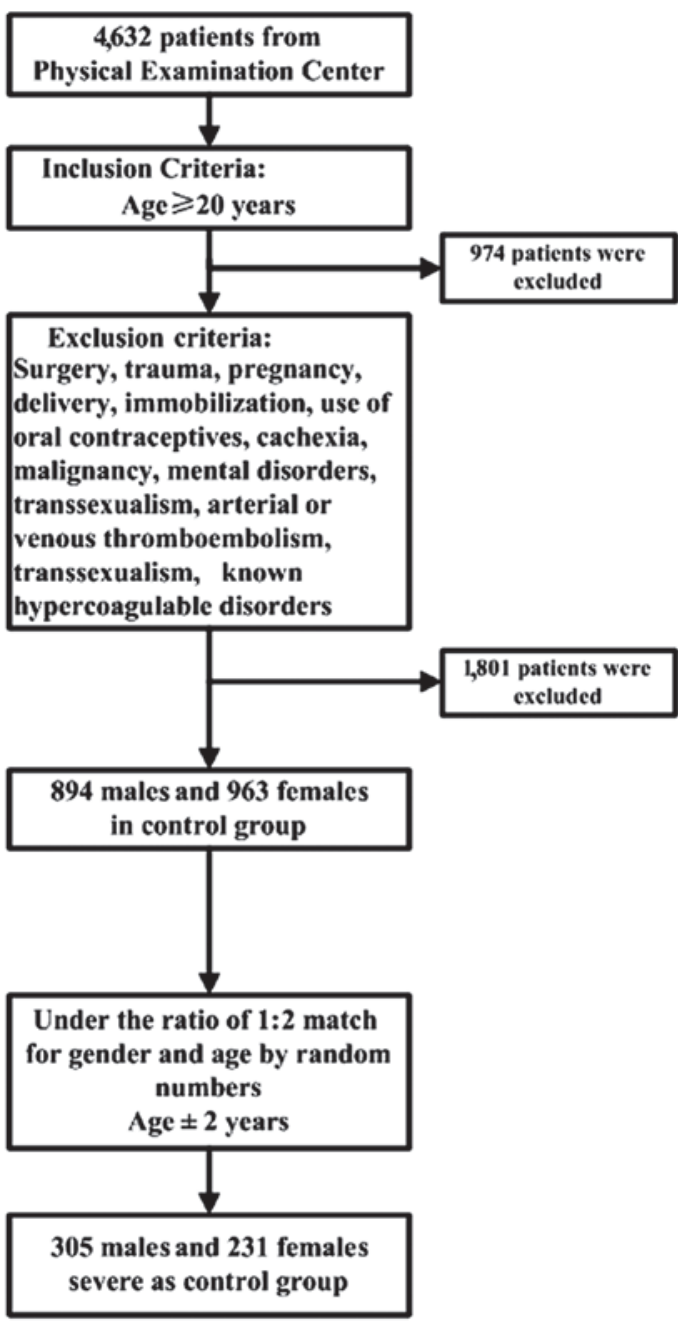

Figure 1. Flow diagram of idiopathic VTE and control group patients screening. VTE, venous thromboembolism; SUA, serum uric acid.

Conditional logistic regression was employed to estimate the odds ratios (ORs) and 95\% confidence intervals (CIs) for the association between SUA per-SD $(92.67 \mu \mathrm{mol} / \mathrm{l})$ increase and idiopathic VTE. Univariate analysis was performed to examine the effect of each parameter on idiopathic VTE (Table II).

The association between SUA and idiopathic VTE was examined using the smoothing plot, with an adjustment for gender, age, LDL-C, total cholesterol, FBG, and stratification by high HDL-C (HDL-C $>1.036 \mathrm{mmol} / 1$ for men or $>1.295 \mathrm{mmol} / \mathrm{l}$ for women) (Fig. 2). The interaction analysis was applied to confirm the effect of high HDL-C levels on SUA for idiopathic VTE (Table III). An initial model was adjusted only for matching variables. Additionally, a second model was adjusted for gender, age, LDL-C, total cholesterol, and FBG.

$\mathrm{P}<0.05$ was considered to indicate a statistically significant difference. Statistical analyses were performed using Empower (R) (http://empowerstats.com/en/; X\&Y Solutions, Inc., Boston, MA, USA) and R (https://www.R-project.org).

Ethical issues and confidentiality. The present study was approved by the Medical Ethics Committee of Wuhan Union
Hospital (Hubei, China). Under the provision that no personal identifiers be recorded, approval for using data from medical records was only for retrospective study analysis. Thus, no individual consent was obtained.

\section{Results}

Patient characteristics. A total of 276 patients were included in the idiopathic VTE group and 536 gender- and age-matched subjects were included in the control group. The mean ages in the case and control groups were $52.9 \pm 14.4$ and $52.7 \pm 14.5$ years. Idiopathic VTE patients and controls were Chinese and the basic characteristics of the study population are provided in Table I. There were no statistically significant differences of the study population in baseline regarding age, gender, SUA, triglyceride, current smoking, and hypertension. The number of thrombotic events was higher in the male (158 males) than in the female (118 females) participants. The sites of VTE were 189 patients $(68.48 \%)$ in the left lower extremity, 77 patients $(27.9 \%)$ in the right lower extremity, PE in 33 patients (11.96\%), 7 patients in the two lower extremities, 2 patients in the left upper extremity, 1 patient in the right upper extremity 
Table II. Univariate analysis was performed to examine the influence of each parameter on idiopathic VTE.

\begin{tabular}{lcc}
\hline Characteristics & P-value & OR $(95 \% \mathrm{CI})^{\mathrm{a}}$ \\
\hline $\begin{array}{l}\text { Gender } \\
\text { Male }\end{array}$ & 0.740 & Ref. \\
Female & & $1.59(0.103-24.55)$ \\
Age (years) & 0.842 & $1.01(0.93-1.09)$ \\
SUA per-SD increase & 0.068 & $1.16(0.989-1.36)$ \\
HDL-C (mmol/l) & 0.022 & $0.588(0.374-0.925)$ \\
LDL-C (mmol/l) & $<0.001$ & $1.43(1.17-1.75)$ \\
Triglyceride (mmol/l) & 0.277 & $1.10(0.93-1.29)$ \\
Total cholesterol (mmol/l) & 0.005 & $1.25(1.07-1.46)$ \\
FBG (mmol/l) & $<0.001$ & $1.24(1.12-1.37)$ \\
Current smoker & 0.036 & Ref. \\
No & & $1.49(1.03-2.15)$ \\
Yes & & Ref. \\
Hypertension & 0.900 & $1.030(0.674-1.57)$ \\
No & & \\
Yes & &
\end{tabular}

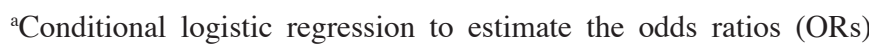
and $95 \%$ (CIs) for the association between each parameter and idiopathic VTE. HDL-C, high-density lipoprotein cholesterol; LDL-C, low-density lipoprotein cholesterol; VTE, venous thromboembolism; SUA, serum uric acid; FBG, fasting blood glucose; CI, confidence interval; Ref., reference.

and inferior vena cava thrombosis in 1 patient. This result also confirmed the finding that the general population usually develop DVT in the left lower limb due to right common iliac artery compression anatomically.

Systemic findings associated with idiopathic VTE. The univariate analysis revealed that HDL-C, LDL-C, total cholesterol, FBG, and current smoking status were significantly associated with idiopathic VTE (Table II). HDL-C showed a negative correlation with the increased risk of idiopathic VTE, a strong protective factor $[\mathrm{P}=0.022, \mathrm{OR}=0.588(95 \% \mathrm{CI}$ : 0.374-0.925)]. Additionally, LDL-C and total cholesterol were positively correlated with idiopathic VTE, $\mathrm{P}<0.001, \mathrm{OR}=1.43$ (95\% CI: 1.17-1.75); $\mathrm{P}=0.005, \mathrm{OR}=1.25$ (95\% CI: 1.07-1.46), respectively. In addition to recognized atherosclerosis risk factors, hyperglycemia and current smoking status presented a similar close association with the disease, $\mathrm{P}<0.001, \mathrm{OR}=1.24$ (95\% CI: 1.12-1.37); $\mathrm{P}=0.036, \mathrm{OR}=1.49$ (95\% CI: 1.03-2.15). In the univariate analysis, we did not identify any correlations of SUA per-SD increase $[\mathrm{P}=0.068, \mathrm{OR}=1.16(95 \% \mathrm{CI}$ : $0.989-1.36)]$, triglyceride $[\mathrm{P}=0.277, \mathrm{OR}=1.10(95 \% \mathrm{CI}$ : $0.93-1.29)]$, and hypertension $[\mathrm{P}=0.900, \mathrm{OR}=1.030$ (95\% CI: 0.674-1.57)] to idiopathic VTE.

Interaction analysis for SUA and HDL-C in Idiopathic VTE. To determine whether an irrelevant relationship of SUA and idiopathic VTE was caused by the interactions among other

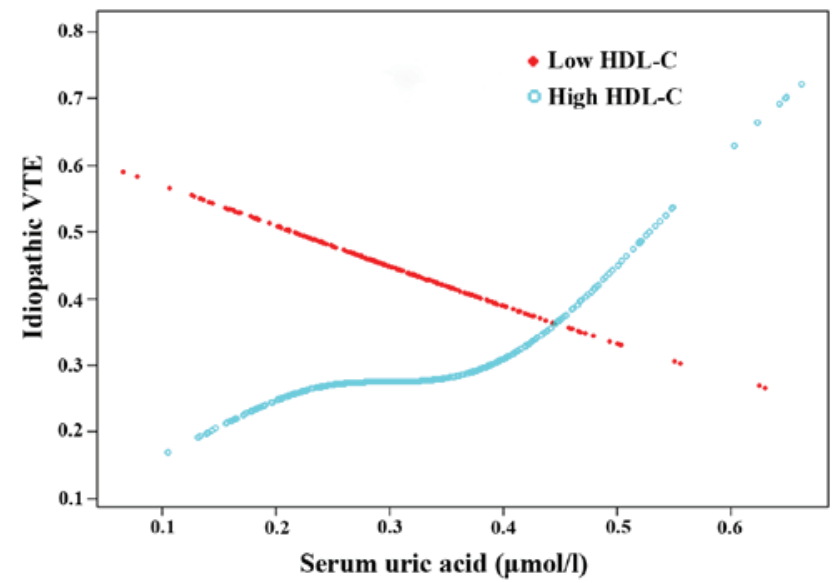

Figure 2. The relationship between serum uric acid and risk of idiopathic VTE by the smoothing plot, with an adjustment for gender, age, LDL-C, total cholesterol, FBG, and stratification by high HDL-C. HDL-C, high-density lipoprotein cholesterol; VTE, venous thromboembolism.

risk factors, we conducted the interaction analysis to scan for interaction factors. After checking for each parameter, we found only the interaction of HDL-C to be significant $(\mathrm{P}<0.001$ for interaction), but not for age, gender, FBG, LDL-C and total cholesterol.

HDL-C was stratified into the high and low groups (men, $<1.036 \mathrm{mmol} / 1$ and women, $<1.295 \mathrm{mmol} / \mathrm{l}$ ) to examine its interaction with SUA for idiopathic VTE (Table III). In the total HDL-C population, there was no obvious association between SUA and idiopathic VTE. However, following stratification of HDL-C into two groups, a significant association of SUA to idiopathic VTE was identified in the high HDL-C population ( $\mathrm{P}=0.003$ for crude and $\mathrm{P}=0.032$ for adjusted), while this correlation was not observed in the low HDL-C population (Fig. 2).

\section{Discussion}

In this retrospective case-control study, we determined the value of SUA, HDL-C, LDL-C, total cholesterol, triglyceride, FBG, current smoking and hypertension for the correlation of idiopathic VTE. The results showed that there were a close association of HDL-C, LDL-C, total cholesterol, FBG and current smoking with the disease. Notably, the association between SUA and idiopathic VTE varied with the HDL-C level, in which SUA was significantly associated with idiopathic VTE at a high HDL-C level, while the correlation was no longer present when the HDL-C level was decreased.

The effect of HDL-C on the risk of VTE is not as obvious as that for arterial thrombosis. However, previous findings revealed an association between $\operatorname{HDL}$ and VTE $(3,10)$. According to size and density, HDL can be classified into the subcategories: i) HDL3, smaller with a high density of $1.125-1.21 \mathrm{~g} / \mathrm{ml}$; and ii) HDL2, larger with a low density of $1.063-1.125 \mathrm{~g} / \mathrm{ml}$, and each subgroup can be subdivided by their apolipoprotein (apo) composition. Generally, HDL2 are apoA-I- and apoE-rich particles, while HDL3 are apoA-Iand apoA-II-rich but apoE less particles (3). It has been suggested that the HDL that is enriched in apoA-I may be more cardioprotective than apoA-I/-II-dominated HDL; therefore, 
Table III. Association of SUA per-SD increase with the risk of idiopathic VTE in different HDL-C levels.

\begin{tabular}{|c|c|c|c|c|c|}
\hline \multirow[b]{2}{*}{ Model } & \multicolumn{2}{|c|}{ Low HDL-C } & \multicolumn{2}{|c|}{ High HDL-C } & \multirow[b]{2}{*}{$\mathrm{P}$ for interaction } \\
\hline & OR $(95 \% \mathrm{CI})$ & P-value & OR $(95 \% \mathrm{CI})$ & P-value & \\
\hline Model 1 & $0.865(0.522-1.43)$ & 0.574 & $1.39(1.12-1.73)$ & 0.003 & 0.0040 \\
\hline Model 2 & $0.852(0.513-1.42)$ & 0.538 & $1.38(1.11-1.72)$ & 0.004 & 0.0041 \\
\hline Model 3 & $0.813(0.471-1.40)$ & 0.455 & $1.29(1.02-1.64)$ & 0.032 & 0.0026 \\
\hline
\end{tabular}

Model 1, conditional logistic regression model not adjusted; model 2, conditional logistic regression model adjusted for matching variables; model 3, conditional logistic regression model adjusted for matching variables, LDL-C, total cholesterol and FBG. High HDL-C, HDL-C >1 .036 $\mathrm{mmol} / \mathrm{l}$ for men or $>1.295 \mathrm{mmol} / \mathrm{l}$ for women. HDL-C, high-density lipoprotein cholesterol; LDL-C, low-density lipoprotein cholesterol; VTE, venous thromboembolism; SUA, serum uric acid; SD, standard deviation; CI, confidence interval.

HDL2 may have an advantage over HDL3 on the protective effects of the cardiovascular system (11). Deguchi et al (5) found that in the DVT patients with or without PE, the HDL2, HDL-C and apoA-I levels were distinctly low. Correspondingly, a subsequent study of VTE indicated that for each increase of $0.1 \mathrm{mg} / \mathrm{ml}$ plasma apoA-I, there would be a 0.87 (95\% CI: 0.80-0.94) relative risk of VTE recurrence (10). Furthermore, the antithrombolic function of HDL enhances the anticoagulant protein $\mathrm{C}$ pathway to attenuate the expression of the tissue factor and reduce thrombin generation, and promotes the synthesis of prostacyclin (12). Additionally, the size of HDL-C and HDL was negatively correlated with the plasminogen activator inhibitor-I level, an inhibitor of tissue plasminogen and plasminogen activators, indicating that HDL particles may be involved in the promotion of plasmin generation and fibrinolysis to reduce the occurrence of thrombotic events (13).

As the end product of purine, SUA has been investigated to be closely associated with CVD. Numa et al (14) identified that for the nonvalvular atrial fibrillation patients, the SUA level was considered an independent indicator of transesophageal echocardiography risk, which includes thrombi and aortic atherosclerosis. Tang et al (15) also reached a similar conclusion whereby the hyperuricemia was a modest predictor for left atrial thromboembolism occurrence among nonvalvular atrial fibrillation patients. The mechanisms of SUA damage for CVD are considered mainly as oxidative stress and inflammation. Some investigators suggested that there are three mechanisms that may induce UA injury to the vessel endothelium: i) Aggravated inflammatory reaction in endothelial cells and proliferation in vascular smooth muscle cells as a consequence of active oxygen species produced in UA metabolism; ii) UA crystals that stimulate the production of multinucleate cells and inflammation mediators leading to damage in the vascular endothelium; and iii) UA is transported into vascular endothelial and smooth muscle cells to trigger the cascade reaction leading to fetal intracellular damages (16). The above mechanisms partially explained the association between SUA and endothelium injury, which is an essential aspect in Virchow's triad for VTE formation.

Recently, Zhang et al (17) conducted a study in coronary artery disease (CAD) patients on the association between SUA level and lipoprotein subfractions, which are considered promising new measurements of CAD risk assessment on blood plasma lipids. From their study, the SUA level was confirmed to be correlated with small dense LDL-C positively and large HDL-C negatively, and this correlation may have an impact on the risk prediction of SUA for CAD to a certain extent. In addition, it has been suggested that compared with the quantity, the quality of subfractions is more important; thus, a decreased large HDL-C may be more atherogenic although the entire HDL-C level remains normal (18).

In the present study, HDL-C manifested a strong protective effect on the disease, and the correlation of SUA and idiopathic VTE was only detected in the high HDL-C population. This is likely due to SUA attenuating large HDL-C quality albeit its total amount of HDL-C remains at a high level and its correlation to idiopathic VTE was obvious under the specific conditions. However, for the low HDL-C population, the decreased number of HDL-C has become a confounding factor and the relationship between SUA and idiopathic VTE was not distinct in this population.

Additionally, it has been suggested that the excessively produced or decreased clearance of UA is a potential etiology of metabolic syndrome and type 2 diabetes mellitus (19), in which the inflammation markers are correlated with an increased number and activity of platelets and fibrinogen, leading to a prothrombotic state. Those findings also indicated that the overburdened UA in hypertension is as critical as insulin resistance, contributing to circulating insulin accumulation and the acceleration of UA intracellular transportation and resorption (20). Considering the interaction of SUA, HDL-C and blood glucose in the disease, we hypothesize that the controversial results obtained in previoius studies on the association of metabolic syndrome and idiopathic VTE was caused by SUA leading to different degrees of HDL-C reduction. As for the number of metabolic syndrome patients, their HDL-C remained at a normal level, although the protective function, and quality of large HDL-C, was already weakened by SUA and these patients were prone to develop VTE events. Therefore, taking total HDL-C as a risk factor for idiopathic VTE, and not distinguishing its levels or components, may cause the interaction, leading to inconsistent conclusions. To the best of our knowledge, few studies considered SUA as s risk factor for idiopathic VTE and none assessed the interaction analysis between HDL-C and SUA in the disease.

Although the harmful role of smoking in arterial thrombosis is well-established, its effect on venous thrombus remains to be determined. The currently known adverse effect 
of smoking on the vascular system is mainly on endothelial damage which may up-regulate the vessel prothrombotic and pro-inflammatory states, disturb platelet function, damage the vascular wall by increasing inflammatory mediators, and reduce the endothelial NO production to make hemodynamic changes (21). Sweetland et al (22) conducted a large prospective study of women with a smoking habit in England, and the results showed that the current smokers had a significantly increased incidence of VTE compared with never-smokers, and the risk was more significant in heavier compared to lighter smokers. However, Enga et al (23) who carried out the Troms $\varnothing$ study concluded that heavy smoking is a risk factor only limited to provoking VTE. When taking cancer and myocardial infarction into account, the obvious association was no longer present, suggesting that the risk of VTE was altered by the development of malignancy or myocardial infarction. Another two large population-based case control studies $(24,25)$ were in agreement with the results of the Troms $\varnothing$ study whereby smoking itself was a weak risk factor and did not significantly impact the development of idiopathic VTE. Our results corroborate the previous finding regarding an association between smoking and idiopathic VTE. At the same time, our results were consistent with those of the study on longitudinal investigation of thromboembolism etiology (7), in which hypertension was not relevant to VTE.

Thus, SUA may act as an important connection between atherosclerosis and idiopathic VTE by influencing the functional HDL-C and it should be considered a critical loop in the progress of pro-inflammatory and pro-thrombotic state in the disease. Taking the role of hyperuricemia, dyslipidemia and hyperglycemia in the pathogenesis of VTE together, it is reasonable to consider idiopathic VTE as a consequence of metabolic disorders.

There are some limitations to the present study. Firstly, diagnosed cancer patients were excluded from the study, albeit some patients with idiopathic VTE may have had occult cancer during the time of investigation since not all the patients underwent screening of tumor markers. The effect of occult cancer on the dyslipidemia, hyperglycemia and hyperuricemia is unknown while its impact on the results of our analysis is probably low. Secondly, since idiopathic VTE patients were defined without using the most common known risk factors, thrombophilia (such as Factor V Leiden) and some heterogeneity (such as prothrombin G20210A mutation) were not investigated in the present study. However, this is unlikely to have had a significant influence on our results as there is currently no exact evidence suggesting that hypercoagulation would result in dyslipidemia, hyperglycemia or hyperuricemia (26). Since this is a retrospective study, the patients in our control group enrolled according to their detailed history but not the performance of ultrasound or CTA to screen out asymptomatic VTE, which may not appropriately represent a generally healthy population. However, the current prevalence of dyslipidemia, hyperglycemia and hyperuricemia in the control group were slightly higher than that reported in the general Chinese population (27-29), indicating that following selection of a different control group the ORs may have been even greater than those reported in the current study.

In conclusion, our findings suggest that a decreased HDL-C level, increased total cholesterol, FBG, and current smoking are closely associated with idiopathic VTE. SUA was associated with increased risk of idiopathic VTE in the high HDL-C population, which may act as a pivotal connection between atherosclerosis and venous thrombus. However, additional studies are to be conducted to confirm these findings.

\section{Acknowledgements}

The present study was supported by the National Natural Science Foundation of China (grant no. 81270391). The investigators are grateful to Professor Sun Yi, Dr Chen Xinglin, Dr Yang Chong and Dr Chen Qijun for their invaluable assistance and advice.

\section{References}

1. Kearon C: Epidemiology of venous thromboembolism. Semin Vasc Med 1: 7-26, 2001.

2. Heit JA: Venous thromboembolism epidemiology: Implications for prevention and management. Semin Thromb Hemost 28 (Suppl 2): 3-13, 2002.

3. van der Stoep M, Korporaal SJ and Van Eck M: High-density lipoprotein as a modulator of platelet and coagulation responses. Cardiovasc Res 103: 362-371, 2014.

4. Brækkan SK, Hald EM, Mathiesen EB, Njølstad I, Wilsgaard T, Rosendaal FR and Hansen JB: Competing risk of atherosclerotic risk factors for arterial and venous thrombosis in a general population: The Tromso study. Arterioscler Thromb Vasc Biol 32: 487-491, 2012.

5. Deguchi H, Pecheniuk NM, Elias DJ, Averell PM and Griffin JH: High-density lipoprotein deficiency and dyslipoproteinemia associated with venous thrombosis in men. Circulation 112: 893-899, 2005.

6. Ageno W, Becattini C, Brighton T, Selby R and Kamphuisen PW: Cardiovascular risk factors and venous thromboembolism: A meta-analysis. Circulation 117: 93-102, 2008.

7. Glynn RJ, Danielson E, Fonseca FA, Genest J, Gotto AM Jr, Kastelein JJ, Koenig W, Libby P, Lorenzatti AJ, MacFadyen JG, et al: A randomized trial of rosuvastatin in the prevention of venous thromboembolism. N Engl J Med 360: 1851-1861, 2009.

8. Steffen LM, Cushman M, Peacock JM, Heckbert SR, Jacobs DR Jr, Rosamond WD and Folsom AR: Metabolic syndrome and risk of venous thromboembolism: Longitudinal investigation of thromboembolism etiology. J Thromb Haemost 7: 746-751, 2009.

9. Zhao G, Huang L, Song M and Song Y: Baseline serum uric acid level as a predictor of cardiovascular disease related mortality and all-cause mortality: A meta-analysis of prospective studies. Atherosclerosis 231: 61-68, 2013.

10. Eichinger S, Pecheniuk NM, Hron G, Deguchi H, Schemper M, Kyrle PA and Griffin JH: High-density lipoprotein and the risk of recurrent venous thromboembolism. Circulation 115: 1609-1614, 2007

11. Umaerus M, Rosengren B, Fagerberg B, Hurt-Camejo E and Camejo G: HDL2 interferes with LDL association with arterial proteoglycans: A possible athero-protective effect. Atherosclerosis 225: 115-120, 2012.

12. Mineo C, Deguchi H, Griffin JH and Shaul PW: Endothelial and antithrombotic actions of HDL. Circ Res 98: 1352-1364, 2006.

13. Asselbergs FW, Williams SM, Hebert PR, Coffey CS, Hillege HL, Navis G, Vaughan DE, van Gilst WH and Moore JH: Gender-specific correlations of plasminogen activator inhibitor-1 and tissue plasminogen activator levels with cardiovascular disease-related traits. J Thromb Haemost 5: 313-320, 2007.

14. Numa S, Hirai T, Nakagawa K, Ohara K, Fukuda N, Nozawa T and Inoue H: Hyperuricemia and transesophageal echocardiographic thromboembolic risk in patients with atrial fibrillation at clinically low-intermediate risk. Circ J 78: 1600-1605, 2014.

15. Tang RB, Dong JZ, Yan XL, Du X, Kang JP, Wu JH, Yu RH, Long DY, Ning M, Sang CH, et al: Serum uric acid and risk of left atrial thrombus in patients with nonvalvular atrial fibrillation. Can J Cardiol 30: 1415-1421, 2014.

16. Feig DI, Kang DH and Johnson RJ: Uric acid and cardiovascular risk. N Engl J Med 359: 1811-1821, 2008. 
17. Zhang Y, Xu RX, Li S, Zhu CG, Guo YL, Sun J and Li JJ: Lipoprotein subfractions partly mediate the association between serum uric acid and coronary artery disease. Clin Chim Acta 441: 109-114, 2015

18. Martin SS, Jones SR and Toth PP: High-density lipoprotein subfractions: Current views and clinical practice applications. Trends Endocrinol Metab 25: 329-336, 2014.

19. NakagawaT,HuH,ZharikovS,TuttleKR,ShortRA,GlushakovaO, Ouyang X, Feig DI, Block ER, Herrera-Acosta J, et al: A causal role for uric acid in fructose-induced metabolic syndrome. Am J Physiol Renal Physiol 290: F625-F631, 2006.

20. Zapolski T, Waciński P, Kondracki B, Rychta E, Buraczyńska MJ and Wysokiński A: Uric acid as a link between renal dysfunction and both pro-inflammatory and prothrombotic state in patients with metabolic syndrome and coronary artery disease. Kardiol Pol 69: 319-326, 2011.

21. Barua RS and Ambrose JA: Mechanisms of coronary thrombosis in cigarette smoke exposure. Arterioscler Thromb Vasc Biol 33: $1460-1467,2013$

22. Sweetland S, Parkin L, Balkwill A, Green J, Reeves G and Beral V; Million Women Study Collaborators: Smoking, surgery, and venous thromboembolism risk in women: United Kingdom cohort study. Circulation 127: 1276-1282, 2013.

23. Enga KF, Braekkan SK, Hansen-Krone IJ, le Cessie S, Rosendaal FR and Hansen JB: Cigarette smoking and the risk of venous thromboembolism: The Troms $\varnothing$ Study. J Thromb Haemost 10: 2068-2074, 2012.
24. Blondon M, Wiggins KL, McKnight B, Psaty BM, Rice KM, Heckbert SR and Smith NL: The association of smoking with venous thrombosis in women. A population-based, case-control study. Thromb Haemost 109: 891-896, 2013.

25. Blondon M, Wiggins KL, Van Hylckama Vlieg A, McKnight B, Psaty BM, Rice KM, Heckbert SR and Smith NL: Smoking, postmenopausal hormone therapy and the risk of venous thrombosis: A population-based, case-control study. Br J Haematol 163: 418-420, 2013

26. Woodward M, Lowe GD, Rumley A, Tunstall-Pedoe H, Philippou H, Lane DA and Morrison CE: Epidemiology of coagulation factors, inhibitors and activation markers: The Third Glasgow MONICA Survey. II. Relationships to cardiovascular risk factors and prevalent cardiovascular disease. Br J Haematol 97: 785-797, 1997.

27. Sun GZ, Li Z, Guo L, Zhou Y, Yang HM and Sun YX: High prevalence of dyslipidemia and associated risk factors among rural Chinese adults. Lipids Health Dis 13: 189, 2014.

28. Yang W, Lu J, Weng J, Jia W, Ji L, Xiao J, Shan Z, Liu J, Tian H, Ji Q, et al; China National Diabetes and Metabolic Disorders Study Group: Prevalence of diabetes among men and women in China. N Engl J Med 362: 1090-1101, 2010.

29. Gao B, Zhou J, Ge J, Zhang Y, Chen F, Lau WB, Wan Y, Zhang N, Xing Y, Wang L, et al: Association of maximum weight with hyperuricemia risk: A retrospective study of 21,414 Chinese people. PLoS One 7: e51186, 2012. 